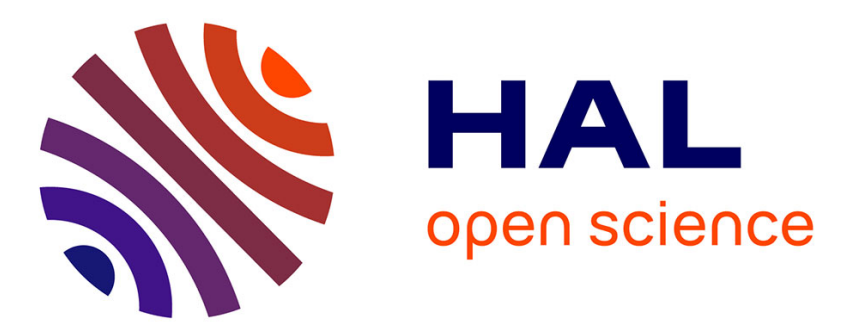

\title{
Wireless sensor network system helping navigation of the visually impaired
}

Kamal Beydoun, Violeta Felea, Hervé Guyennet

\section{To cite this version:}

Kamal Beydoun, Violeta Felea, Hervé Guyennet. Wireless sensor network system helping navigation of the visually impaired. ICTTA'08, IEEE int. conf. on Information and Communication Technologies: from Theory to Applications, 2008, Syria. pp.1-5. hal-00563294

\section{HAL Id: hal-00563294 \\ https://hal.science/hal-00563294}

Submitted on 4 Feb 2011

HAL is a multi-disciplinary open access archive for the deposit and dissemination of scientific research documents, whether they are published or not. The documents may come from teaching and research institutions in France or abroad, or from public or private research centers.
L'archive ouverte pluridisciplinaire HAL, est destinée au dépôt et à la diffusion de documents scientifiques de niveau recherche, publiés ou non, émanant des établissements d'enseignement et de recherche français ou étrangers, des laboratoires publics ou privés. 


\title{
Wireless sensor network system helping navigation of the visually impaired
}

\author{
Kamal Beydoun, Violeta Felea, Hervé Guyennet \\ University of Franche-Comté, LIFC \\ Besançon, France \\ \{beydoun,felea,guyennet\}@lifc.univ-fcomte.fr
}

\begin{abstract}
Navigation aids for the pedestrians are considered new research challenges. The visually impaired are particularly concerned, because of their need to detect and avoid obstacles, as well as to orient themselves in unknown environments. In this paper, we discuss some existing projects in this domain and we propose a novel aid system formed of wireless sensors, meant to help navigation of the visually impaired, which addresses both orientation and obstacle negotiation. The proposed system is GPS-free and does not need external assistance in navigation.
\end{abstract}

Keywords : Wireless Sensor Networks, Navigation aids, energy conservation, zone partitionning, routing protocol.

\section{INTRODUCTION}

The orientation and navigation in unknown environments are old challenges. Orientation is the possibility of locating specific targets and of constructing routes to them. This implies the ability to establish and maintain an awareness of one's position in space relatively to landmarks in the surrounding environment and relative to a particular destination. Navigation extends the orientation challenges by the obstacle detection and avoidance. Thus, information on obstacles is taken into account for the route discovery and planning.

Formerly, the sea and the air were the most difficult places where to be directed because they missed visible roads or other references. Moreover, the navigation systems were developed and integrated into several various applications. Recently, navigation systems for the vehicles appeared on the market and due to technological progresses, the equipment became increasingly small and light-weight. Consequently, the navigation systems became universally applicable, therefore useful for the pedestrians. Navigation for pedestrians became a challenge of research since mobility for people is a very significant component for our modern society.

The navigation systems for the pedestrians are currently at the beginning of their development. Many researches were carried out or are currently work in progress. Pedestrian navigation covers a large field of applications with very different characteristics because the user's needs may vary considerably. One of these needs is the navigation for the visually impaired people and especially for blind individuals.
Existing electronic travel aids (ETAs) for the visually impaired respond either to the need of obstacle detection and avoidance, or to that of spatial orientation. Integration of both aspects in the system design, in order to offer navigation aids, still remains a research challenge.

Our proposed system is meant to help navigation (so both obstacle detection and orientation) of the blind into an urban space equipped with wireless sensors. The blind person is also equipped with an advanced wireless sensor. Sensors form a network enabling transmission of environmental information (obstacles, targets) to the smart sensor which is treating the information, in order to construct the itinerary of the visually impaired. The system is designed to integrate detection of both dynamic and static obstacles. The main advantage of our system is that it does not need any GPS (Global Positioning System) information.

GPS enables mobility when trying to reach a specific destination, which is important for any traveler, the visually impaired included. In spite of its orientation indications, the GPS presents some drawbacks. GPS system can provide accurate information to a traveler if it receives a signal from at least four satellites. Inclement weather and tall buildings can both interfere with signal reception. Another disadvantage of GPS is its dependence on battery power. Even though using a battery makes GPS systems portable, a battery can fail without warning. Moreover, most of the GPS products use digital commercial mapping, and maps become obsolete if not updated periodically. Another drawback is that GPS systems lack in detection of minor changes in the environment. A GPS receiver cannot inform a blind traveler of a drop-off on the sidewalk or a rise in elevation of the ground; therefore a blind pedestrian will not be able to use GPS to determine ordinary obstacles that may lie ahead or to decide whether it is safe to cross the street at a busy crossroad. GPS technology has other disadvantages worth mentioning. The largest drawback is that the GPS system is not reliable in all places where blind people want to travel (e.g. in indoor environments).

\section{ARCHITECTURES AND FUNCTIONALITIES FOR THE VISUALLY IMPAIRED}

The visually impaired requires information on their surroundings when they engage in travels. They need to detect the presence, location and nature of obstacles around them (obstacle negotiation) as well as the presence of particular targets or locations, identified by landmarks (orientation). 
Obstacles may be stationary (objects, buildings, holes, stairs, and floors) or mobile (vehicles, other pedestrians). They may also be classified as underground, on the floor or suspended. Traditional mechanical mobility aids for obstacle detection and avoidance are the long cane and the guide dog. These tools do not offer enough range of detection (the cane), do not identify suspended obstacles (at head-height), are difficult to train or to look after (the guide dog) and are handheld devices; as a result they limit certain actions. In order to offer a maximum degree of safety and independence, electronic mobility aids have been designed. Their aim is to offer the visually impaired the ability to walk naturally and increase their mobilityas well as their travel speed.

Electronic obstacles negotiators (Mowat [1], NavBelt [2], GuideCane [3]) give a larger vision of the 3D environment, using ultrasonic sensors, image sensors or infrared ones, or even standard cameras, which enlarge the cone vision up to 120 degrees. The interface between the electronic aid system and the human makes use of different senses (tactile or auditory).

One important obstacle for the visually impaired in improving their quality of life is the inability to orient themselves. This handicap doesn't give them easy access to buildings, to important facilities (train stations, cultural places, post offices), and limits their use of public transportation. Persons having a visual handicap need to locate targets (destinations) and to construct routes to them. This capacity requires establishing and maintaining awareness of one's spatial position relatively to the destination. Electronic orientation aids may help the visually impaired to orient themselves in unknown environments. Generally, these devices provide information prior to travels (no dynamic information is integrated). MoBIC [4] is a project focused on the aid of the blind in mobility. The MoBIC travel aid consists of two interrelated components: the MoBIC Pre-Journey System (MoPS) to assist users in planning journeys, and the MoBIC Outdoor System (MoODS) to execute these plans by providing users with orientation assistance during journeys. The MoODS is a secondary aid, complementing primary aids such as the long cane or guide dog. It uses derived GPS information.

Authors in [5] describe another GPS-based system, meant for orientation. They propose a module called PNM (Pedestrian Navigation Module) [6]. PNM, in urban canyons with poor GPS signal reception, changes positioning strategy and uses step-length models and algorithms to determine the user's position.

Some of the previous cited aids give limited useful information or require extensive training in the use of the obstacle or orientation map interface.

The integration of obstacle detection within the orientation process helps to take into account the obstacle information in route discovery and planning. Electronic navigation aids, integrating location and orientation with respect to obstacles ahead, are less developed in the current approaches.

Out of few projects proposing navigation aids (so both obstacle detection and orientation) for the visually impaired is the remote sighted guidance system developed at Brunel University. Its architecture is illustrated in Fig. 1.

This system integrates three main technologies: Global Positioning System (GPS), Geographical Information System (GIS) database and digital video streaming over 3rd Generation (3G) mobile networks [7,8].

The system user contacts, firstly, the guide and requests navigation assistance from the Navigation Service Centre. During user displacement, the video camera captures video images of the surrounding and the GPS receiver gives the position. This data is sent to the guide. By using the GIS module, the guide determines the optimal route in order to arrive to the destination. According to the updated and received information from GPS and camera, the guide communicates navigational instructions to the user [12].

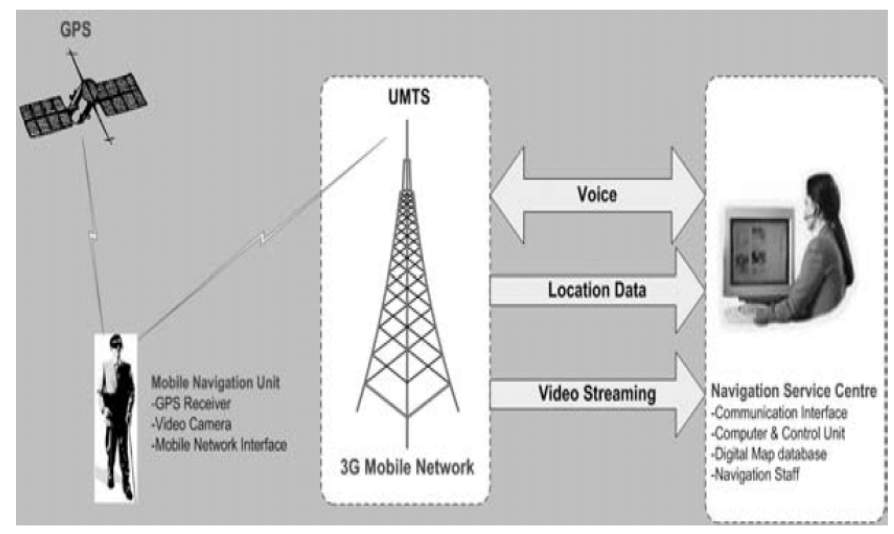

Figure 1. The Brunel University’s system components

The challenges in ETAs research are both technological (finding new sensors to better detect obstacles, new architectures able to reconstruct needed information, new techniques to display the computed information and to give navigation indications to the visually impaired) and functional (associating obstacle identification and space orientation).

Our objective is to propose a new wireless sensor network system deployed on a large scale based on current and low cost technology, which enables the navigation of the visually impaired both indoors and outdoors. The aim of this system is offering mobility for the visually impaired in their journeys, and a maximum degree of independence. The former objective is achieved through the smart sensor worn by the visually impaired, collecting information about the environment. The latter exploits sensed information from devices placed in the surroundings. The deployment of these devices ensures, unlike GPS technology, that information on the environment can always be extracted. The general description of this system is presented in the next section, with the scientific challenges involved.

\section{THE PROPOSED NAGIVATION SYSTEM}

We propose a system based on a wireless sensor network architecture deployed on an urban space, in which sensors communicate (see Fig. 2) and transmit information to a particular smart sensor, worn by the visually impaired person. In order to guide the visually impaired, the sensing sensors 
collect information about the static obstacles (i.e. wall, barrier...) and the dynamic obstacles (i.e. cars, people...). The person to be guided is equipped with a smart sensor that treats and translates the information collected by the sensing sensors, and also interacts with the network.

In the following, we pose a scenario to exemplify the approach. When the visually impaired interrogates, via the smart sensor, the sensor network in order to find a route to a particular destination, sensors collaborate to find the best routes, taking into account static obstacles and distance factors.

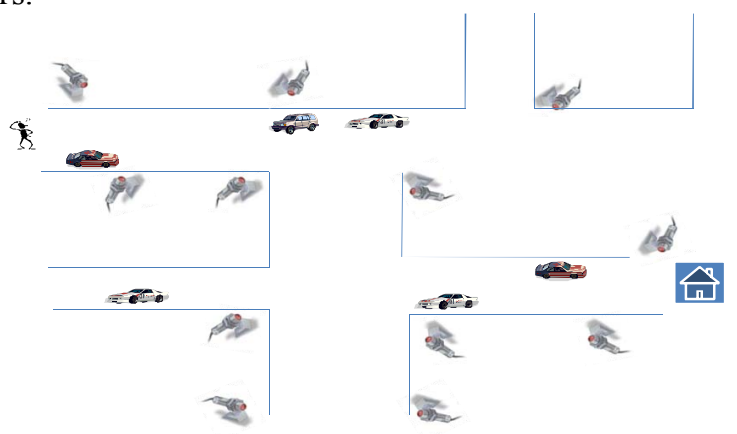

Figure 2. Sensors deployed on the urban space

A route is formed of a sequence of sensors identifiers, which helps to daw a navigation map for the visually impaired. During navigation, dynamic information on the environment is transmitted to the person, like the presence of vehicles in motion (see Fig. 3). The itinerary may, consequently, be modified according to these data.

Two main scientific challenges are dealt with in this architecture and system: system lifetime and routing protocols (the process of selecting paths in a network along which data is sent).

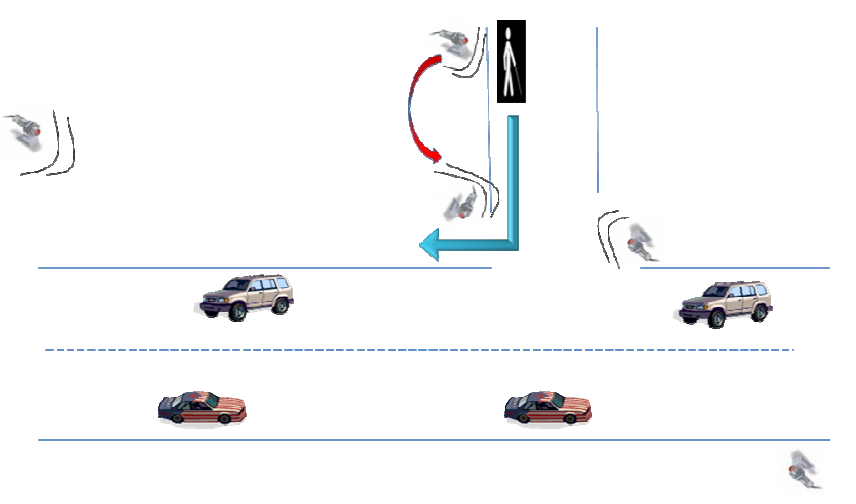

Figure 3. Communication between sensors

The energy of sensors in the network is essential to preserve the total lifetime of the system. During navigation of the visually impaired, several zones are not concerned with his travel, so sensors in those areas may be put into the sleep mode. This behavior led us to propose an algorithm of sensor organization, which divides the network into zones. In this way, only the sensors of the sub-zone in which the person moves are in communication with him. Therefore, the sensors in the other zones are switched off into the sleep mode in order to save their energy. We will detail this algorithm in the next section.

A second research objective is finding routing protocols based on this architecture. The energy issue is also integrated in the routing approaches, making routing decision inside a zone and between zones (intra and inter zone routing protocols).

\section{ZONE PARTITIONING ALGORITHM}

In this section we will briefly describe the new approach for sensors grouping in multiple zones. Firstly we give the objectives. Zone partitioning helps with addressing the scalability and energy efficiency issues in large sensor networks which is the case for our deployed sensor infrastructure. Classical ad-hoc routing in large wireless sensor networks is too expensive because of the routing table space storage and processing. Routing in smaller groups of sensor uses reduced routing tables, but needs another routing protocol between zones. Routing does not use specific zone node, as the cluster heads, in clustering algorithms, because cluster heads are not always reachable in one hop communication. We do not favor particular nodes forwarding task, but we make a two-level routing: between zones and inside zones.

As illustrated in Fig. 4, nodes are grouped in disjoint zones. The nodes of a zone can communicate between each other within one or more hops. If such information must be exchanged between a zone and another, it should pass by the nodes at the border with the other zone (dashed arrows). Our algorithm virtually constructs the zones with the necessary routing information. The border nodes will have a list of the border nodes of the others adjoining zones.

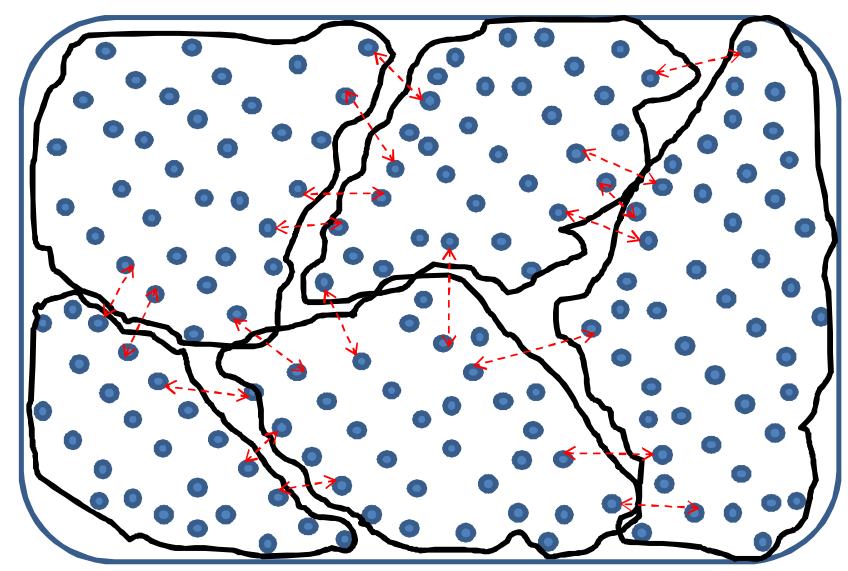

Figure 4. Wireless Sensor Network zone infrastructure

In the suggested approach, the sensors are deployed randomly. We suppose that each node has a global ID. Information concerning nodes (such as energy, link states, geographic positions, etc...) is not required. This algorithm divides a wireless sensor network into several zones and provides useful information for the routing. There are some randomly selected nodes named inviting nodes, which launch the algorithm. 
Zones are determined depending on the zone radius. The zone radius is the maximum number of hops between the inviting node and the invited nodes to its zone. The number of zones and the zone radius are algorithmic parameters that can be modified. The number of inviting nodes equals the number of zones. The detailed algorithm is given in [11].

\section{EXPERIMENTATION}

Experiments were built upon the J-Sim simulator [9] dedicated to WSN simulations. It is a component-based simulation environment, using the notion of autonomous component programming model. J-Sim is developed entirely in Java.

In order to show the performance and the effectiveness of our algorithm, we compared it to another similar group division technique based on the number of hops, that of Lin and Chu [10]. They proposed a clustering algorithm that organizes nodes in clusters based on the number of hops. Each cluster has a cluster head and nodes of a cluster are within a given maximum number of hops $\mathrm{R}$ from the cluster head. Clusters may be dismissed and new clusters may be formed. A cluster is dismissed when two cluster heads are within a distance smaller than a predetermined number of hops $D(D<=R)$, called cluster dismiss distance. In this case, each node of the dismissed cluster will try to join a new cluster. If a node cannot find a cluster head within $\mathrm{R}$ hops it becomes the cluster head of a new cluster. In order to make the clustering, each node has a 4-tuple information $\left(\mathrm{i}, \mathrm{C}_{\mathrm{i}}, \mathrm{D}_{\mathrm{i}}, \mathrm{N}_{\mathrm{i}}\right)$ including:

1. the ID of the node, supposed unique and represented as a positive number,

2. the cluster head ID of the cluster to which the node belongs to,

3. the number of hops from the node to its cluster head, and the ID of the node along the path from the node to its cluster head.

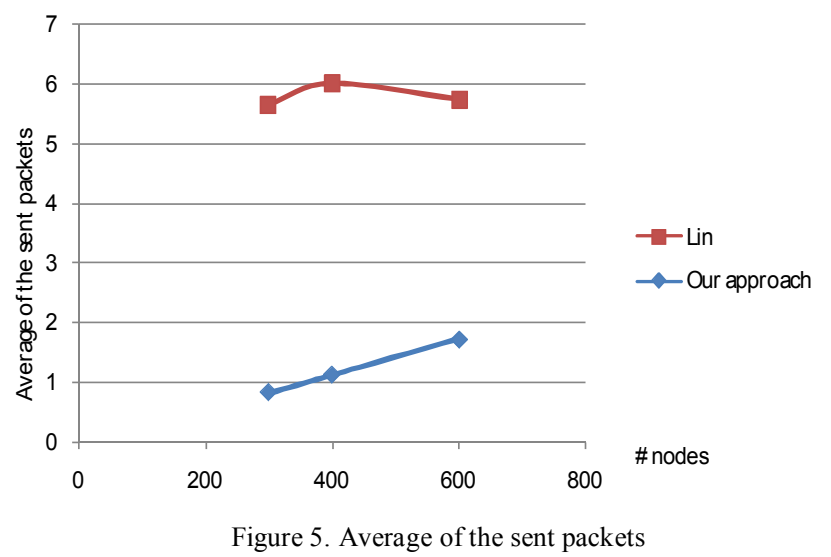

We have demonstrated that our approach is energy-efficient because the number of sent packets is reduced considerably (see Fig. 5). We have also demonstrated the scalability of our approach by increasing the number of nodes in the network. Another advantage of our approach is that we can precise, in advance, the required number of zones $(z N=15$ in these simulations) which is not the case in Lin's algorithm where the number of zones may be very important.
Lin's algorithm creates small zones (several zones contain one/two nodes). Moreover, the number of zones in Lin's algorithm can be neither bounded nor pre-defined. Table I compares the number of zones generated by both algorithms. The error rate, in our approach, is the percentage of the unallotted nodes to any zone (these are alone). We can see that even if some nodes remain unallotted in our approach, their number progressively decreases when the number of nodes increases (Table I). That shows the scalability of the approach. In Lin's algorithm, we have calculated the error rate as the percentage of the nodes that are alone in their zones. As shown in Table I, the error rate is important and increases when the number of nodes increases (or the network density increases). Furthermore, Lin's algorithm generates a considerable number of zones. Each zone has few nodes (in average 2 nodes per zone) which is not adequate for an intrazone routing protocol as intended in our system. Unlike this behavior, in our approach, the number of zones is predefined and the average number of nodes per zone is 26. More simulations and results are shown in [11].

Table I. Error rate and number of zones

\begin{tabular}{ccccc}
\hline \# nodes & \multicolumn{2}{c}{ Our approach } & \multicolumn{2}{c}{ Lin's algorithm } \\
\hline & Error & zN & Error & zN \\
300 & $24 \%$ & 15 & $38,3 \%$ & 164 \\
400 & $8 \%$ & 15 & $34,2 \%$ & 210 \\
600 & $0,7 \%$ & 15 & $46,1 \%$ & 325 \\
\hline
\end{tabular}

\section{CONCLUSION}

This paper presents a novel wireless sensor network system to help the visually impaired during navigation. This navigation method includes approaches that integrate both obstacle detection/avoidance and orientation/guidance aids, thus taking into account information on obstacles in route discovery and planning. It is GPS-free needing only low cost devices and obstacle detection sensor technologies. We also don't need remote assistance to guide the visually impaired. We have also cited other existing systems and aid technologies that are not complete solutions for helping navigation of the visually impaired.

The architectural approach highlights the challenges of minimizing energy consumption and of providing routing protocols. The virtual structure proposed through zone partitioning simplifies routing and can help to reduce energy consumption by switching particular sensors to the sleep mode.

\section{REFERENCES}

[1] N. Pressey, "Mowat Sensor," Focus, vol. 3, pp. 35-39, 1977.

[2] S. Shoval, J. Borenstein, and Y. Koren, "The NavBelt-a computerized travel aid for the blind based on mobile robotics technology," IEEE Trans Biomed Eng., vol. 45, no. 11, pp. 1376-86, 1998.

[3] J. Borenstein and I. Ulrich, "The GuideCane - A Computerized Travel Aid for the Active Guidance of Blind Pedestrians," in Proceedings of the IEEE International Conference on Robotics and Automation, Albuquerque, NM, April 1997, pp. 1283-1288. 
[4] H. Petrie, V. Johnson, T. Strothotte, S. Fritz, R. Michel, and A. Raab, "MoBIC: Designing a Travel Aid for Blind and Elderly People," Journal of Navigation, vol. 29, no. 1, pp. 45-52, 1996.

[5] Q. Ladetto and B. Merminod, "In Step with INS - Navigation for the Blind Tracking Emergency Crews," GPS World - www.gpsworld.com, 2002.

[6] P. Guilliéron, D. Büchel, I. Spassov and B. Merminod, "Indoor Navigation Performance Analysis," in ENC GNSS, Rotterdam, 2004.

[7] V. Garaj, et al., A system for remote sighted guidance of visually impaired pedestrians, British Journal of Visual Impairment 21 (2) (2003) (UK)

[8] Z. Hunaiti: Personal Navigation System using 3rd Generation Mobile Technology in conjunction with Global Positioning System. M.Phil.-Ph.D.
Transfer Report, Systems Engineering Department, Brunel University,London UK (2003).

[9] J-Sim Home page. [Online] 01, 2005. http://www.j-sim.org/

[10] Lin, H., Chu, Y.: A clustering technique for large multihop mobile wireless networks. In: Vehicular Technology Conference Proceedings. Vol. 7, pp. 1545-1549. Tokyo, Japan (2000).

[11] K. Beydoun, V.Felea, H.Guyennet. Energy-Efficient WSN Infrastructure. Submitted to International Workshop on Distributed Collaborative Sensors Networks, Irvine, California, USA, 2008.

[12] Hunaiti Z., Garaj V. and Balachandran W. A Remote Vision Guidance System for Visually Impaired Pedestrians. The journal of navigation (2006), 59, 1-8 